Supporting Information

\title{
A Systematic Cheminformatics Analysis of Functional Groups Occurring in Natural Products
}

Peter Ertt* and Tim Schuhmann

Novartis Institutes for BioMedical Research, $\mathrm{CH}-4056$, Basel, Switzerland

\section{Identification of the source organism class}

When processing NPs from the Dictionary of Natural Products only molecules where the producing organism could be identified were included in the analysis. The NP producing organisms were identified with the help of the Taxonomy Database of the National Center for Biotechnology Information. A Python script was used to analyze the information from the biological source field (BSRC) of the DNP, to find the scientific name if present and to match it to the entry in the taxonomy database. Since this database is organized in a form of a phylogenetic tree it was possible to assign the source organism to one of four classes (animals, plants, fungi or bacteria). If no scientific name could be found the Python script analysed the words in the source description field and compared them with the keywords characteristic for different classes. The following keywords, if found, have been used to assign the origin to one of the four classes:

animals "mammal","human","muscle","animal","fish","liver","kidney","bird","venom","bo vine",,"frog","mouse","pheromone","rat","pig","insect","butterfly","beetle","snake","brain"," snail","mussel"," urine","mollusc","plasma","chicken","toad","bile","coral","sea anemone","sea cucumber"

plants 
"plant","seed","leaf","bark",,"oil","leave","flower","tobacco","seed","juice","resin","root","wi ne","fruit","rhizome","stem","alga","algae","lichen","apple","citrus","coffee","alkaloid","gra pe","soybean","potato","tomato","wood","tea"

\section{fungi}

"fungi","fungus","mushroom","basidiomycete"

\section{bacteria}

"bacter","bacilli","streptomycete"

\section{Calculation of similarity between NPs produced by different organism classes}

The distribution of FGs in NPs produced by different organism classes (animals, plants, fungi or bacteria) has been used to calculate similarity between these four sets. The similarity was calculated as a cosine coefficient between the vectors representing the percentage of $F G$ occurrences in the different classes. The cosine similarity defined as

$$
\text { similarity }=\cos (\theta)=\frac{\mathbf{A} \cdot \mathbf{B}}{\|\mathbf{A}\|\|\mathbf{B}\|}=\frac{\sum_{i=1}^{n} A_{i} B_{i}}{\sqrt{\sum_{i=1}^{n} A_{i}^{2}} \sqrt{\sum_{i=1}^{n} B_{i}^{2}}},
$$

is used often as a measure of similarity between two data sets that may be characterised by vectors of numbers. In this case the vectors $A_{i}$ and $B_{i}$ were the vectors containing the percentage of occurrences of various FGs in the two different sets. These data are available in the file FGinDifferentOrganisms.txt in the Supporting information. The similarity calculated by this procedure is a number between 1 (the two sets have identical distribution of FGs) and 0 (no similarity between the two sets). 
Table S1. Occurrence (in percentage) of functional groups identified in the DNP, the OpenNP and the synthetic molecules (the table may be obtained from the corresponding author upon request as a text file).

\begin{tabular}{|c|c|c|c|}
\hline$F G$ & DNP & OpenNP & synthetic \\
\hline $\mathrm{O}[\mathrm{Cal}]$ & 61.07 & 69.5 & 6.22 \\
\hline $\mathrm{C}=\mathrm{C}$ & 39.85 & 39.8 & 4.04 \\
\hline$[\mathrm{R}] \mathrm{O}[\mathrm{R}]$ & 35.24 & 45.45 & 42.06 \\
\hline$[\mathrm{R}] \mathrm{OC}([\mathrm{R}])=\mathrm{O}$ & 28.65 & 26.01 & 9.01 \\
\hline $\mathrm{O}[\mathrm{Car}]$ & 28.43 & 40.6 & 3.52 \\
\hline$[\mathrm{R}] \mathrm{OCO}[\mathrm{R}]$ & 22.55 & 18 & 2.06 \\
\hline$[R] C([R])=O$ & 15.86 & 20.06 & 3.87 \\
\hline $\mathrm{O}=[\mathrm{Car}]$ & 14.17 & 16.84 & 14.63 \\
\hline [Oar] & 13.6 & 14.08 & 13.25 \\
\hline$[\mathrm{R}] \mathrm{OC}(=\mathrm{O}) \mathrm{C}=\mathrm{C}$ & 11.86 & 8.79 & 0.3 \\
\hline$[R] C(O)=O$ & 10.58 & 14.52 & 4.4 \\
\hline [Nar] & 7.44 & 15.9 & 56.33 \\
\hline$[R] N([R]) C([R])=0$ & 6.73 & 10.16 & 55.97 \\
\hline$[R] C(=O) C=C$ & 5.98 & 9.46 & 0.37 \\
\hline$[R] N([R])[R]$ & 5.66 & 4.77 & 21.49 \\
\hline $\mathrm{C} 1 \mathrm{CO} 1$ & 3.63 & 3.54 & 0.08 \\
\hline $\mathrm{C}=\mathrm{CC}=\mathrm{C}$ & 2.97 & 4.23 & 0.04 \\
\hline$[\mathrm{R}] \mathrm{OCO}$ & 2.51 & 3.57 & 0.03 \\
\hline$[R] O C O C([R])=O$ & 2.47 & 1.65 & 0.02 \\
\hline$[\mathrm{R}] \mathrm{C}=\mathrm{O}$ & 2.43 & 4.39 & 0.27 \\
\hline$[R] N[R]$ & 2.23 & 15.66 & 7.96 \\
\hline $\mathrm{N}[\mathrm{Cal}]$ & 1.85 & 4.19 & 1.14 \\
\hline$[\mathrm{R}] \mathrm{Cl}$ & 1.84 & 1.58 & 18.94 \\
\hline$[\mathrm{R}] \mathrm{Br}$ & 1.45 & 0.31 & 6.32 \\
\hline $\mathrm{OC}(=\mathrm{O}) \mathrm{C}=\mathrm{C}$ & 1.3 & 1.87 & 0.05 \\
\hline$[\mathrm{R}] \mathrm{OS}(\mathrm{O})(=\mathrm{O})=\mathrm{O}$ & 0.97 & 0.24 & 0 \\
\hline $\mathrm{C}=\mathrm{CC}=\mathrm{O}$ & 0.91 & 1.06 & 0 \\
\hline$[R] N([R]) C(=O) C=C$ & 0.78 & 0.79 & 1.1 \\
\hline $\mathrm{C}=[\mathrm{Car}]$ & 0.61 & 2.33 & 4.37 \\
\hline $\mathrm{N}[\mathrm{Car}]$ & 0.56 & 5.53 & 1.87 \\
\hline$[\mathrm{R}] \mathrm{OC}(=\mathrm{O}) \mathrm{C}=\mathrm{CC}=\mathrm{C}$ & 0.56 & 0.42 & 0.01 \\
\hline [Sar] & 0.55 & 0.81 & 17.04 \\
\hline$[\mathrm{R}] \mathrm{N}=\mathrm{C}(\mathrm{N}([\mathrm{R}])[\mathrm{R}]) \mathrm{N}([\mathrm{R}])[\mathrm{R}]$ & 0.55 & 5.2 & 0.11 \\
\hline $\mathrm{C}=\mathrm{CC}(=\mathrm{O}) \mathrm{C}=\mathrm{C}$ & 0.53 & 0.21 & 0.04 \\
\hline$[\mathrm{R}] \mathrm{OCOCO}[\mathrm{R}]$ & 0.49 & 0.36 & 0.01 \\
\hline$[\mathrm{R}] \mathrm{OC}(=\mathrm{O}) \mathrm{C} 1 \mathrm{CO} 1$ & 0.44 & 1.24 & 0.01 \\
\hline$[R] S[R]$ & 0.43 & 0.8 & 9.22 \\
\hline$[\mathrm{R}] \mathrm{OCOCOC}=\mathrm{CC}(=\mathrm{O}) \mathrm{O}[\mathrm{R}]$ & 0.4 & 0.4 & 0 \\
\hline$[\mathrm{R}] \mathrm{OC}=\mathrm{C}$ & 0.39 & 0.56 & 0.03 \\
\hline
\end{tabular}




\begin{tabular}{|c|c|c|c|}
\hline$[R] N([R])([R])=O$ & 0.39 & 0.08 & 0 \\
\hline$[\mathrm{R}] \mathrm{OO}$ & 0.38 & 0.12 & 0 \\
\hline$[\mathrm{R}] \mathrm{C}(=\mathrm{O}) \mathrm{C}=\mathrm{CC}=\mathrm{C}$ & 0.38 & 0.31 & 0.01 \\
\hline [Oar+] & 0.38 & 1.22 & 0 \\
\hline$[\mathrm{R}] \mathrm{N}=\mathrm{C}$ & 0.37 & 0.15 & 0.67 \\
\hline $\mathrm{C \# C}$ & 0.37 & 9.51 & 0.46 \\
\hline $\mathrm{C}=\mathrm{CC}=\mathrm{CC}=\mathrm{C}$ & 0.35 & 1.28 & 0.01 \\
\hline$[\mathrm{R}] \mathrm{OC}(=\mathrm{O}) \mathrm{N}([\mathrm{R}])[\mathrm{R}]$ & 0.35 & 0.24 & 1.27 \\
\hline$[\mathrm{R}] \mathrm{OC}=\mathrm{CC}(=\mathrm{O}) \mathrm{O}[\mathrm{R}]$ & 0.33 & 0.18 & 0.01 \\
\hline$[\mathrm{R}] \mathrm{OCN}([\mathrm{R}])[\mathrm{R}]$ & 0.33 & 1.52 & 0.09 \\
\hline$[\mathrm{R}] \mathrm{OO}[\mathrm{R}]$ & 0.33 & 0.72 & 0 \\
\hline$[\mathrm{R}] \mathrm{OC}=\mathrm{CC}([\mathrm{R}])=\mathrm{O}$ & 0.32 & 0.3 & 0.01 \\
\hline$[\mathrm{R}][\mathrm{N}+]([\mathrm{R}])([\mathrm{R}])[\mathrm{R}]$ & 0.31 & 0.31 & 0.07 \\
\hline$[\mathrm{Nar}+]$ & 0.3 & 2.43 & 0.13 \\
\hline$[\mathrm{R}] \mathrm{C}(=\mathrm{O}) \mathrm{C}=\mathrm{CO}$ & 0.27 & 0.27 & 0.02 \\
\hline$[\mathrm{R}] \mathrm{OC}(\mathrm{O}[\mathrm{R}]) \mathrm{O}[\mathrm{R}]$ & 0.27 & 0.1 & 0 \\
\hline $\mathrm{C}=\mathrm{CC} \# \mathrm{C}$ & 0.27 & 0.15 & 0 \\
\hline$[R] N([R]) C(=O) C=C C=C$ & 0.26 & 0.2 & 0.02 \\
\hline$[\mathrm{R}] \mathrm{N}(\mathrm{C}=\mathrm{C}) \mathrm{C}([\mathrm{R}])=\mathrm{O}$ & 0.26 & 0.15 & 0.04 \\
\hline$[R] N([R]) C=O$ & 0.26 & 0.14 & 0.04 \\
\hline$[\mathrm{R}] \mathrm{OCOCOC}=\mathrm{C}$ & 0.25 & 0.13 & 0 \\
\hline C\#CC\#C & 0.22 & 0.08 & 0 \\
\hline$[R] C(=O) C(O)=C$ & 0.2 & 0.15 & 0 \\
\hline$[\mathrm{R}] \mathrm{N}(=\mathrm{O})=\mathrm{O}$ & 0.2 & 0.21 & 4.74 \\
\hline$[\mathrm{R}] \mathrm{C}(=\mathrm{O}) \mathrm{C} 1 \mathrm{CO} 1$ & 0.2 & 0.17 & 0.01 \\
\hline$[\mathrm{R}] \mathrm{N}([\mathrm{R}]) \mathrm{CO}$ & 0.19 & 1.09 & 0 \\
\hline$[\mathrm{R}] \mathrm{OCOC}=\mathrm{C}$ & 0.19 & 0.1 & 0 \\
\hline$[R] N([R]) C(=O) C(=C) N([R]) C([R])=O$ & 0.19 & 0.31 & 0.12 \\
\hline $\mathrm{CHN}$ & 0.19 & 0.1 & 2.95 \\
\hline $\mathrm{C}=\mathrm{CC} 1 \mathrm{CO} 1$ & 0.19 & 0.1 & 0 \\
\hline$[R] N([R]) C(=O) N([R])[R]$ & 0.17 & 0.18 & 4.51 \\
\hline$[R] N([R]) C N([R]) C([R])=O$ & 0.17 & 0.62 & 0.2 \\
\hline$[\mathrm{R}] \mathrm{N}(\mathrm{CO}) \mathrm{C}([\mathrm{R}])=\mathrm{O}$ & 0.17 & 0.26 & 0.01 \\
\hline$[R] S S[R]$ & 0.17 & 4.66 & 0 \\
\hline$[\mathrm{R}] \mathrm{C}(=\mathrm{O}) \mathrm{C}=\mathrm{CC}([\mathrm{R}])=\mathrm{O}$ & 0.16 & 0.17 & 0 \\
\hline$[\mathrm{R}] \mathrm{OC}(=\mathrm{O}) \mathrm{C}=\mathrm{CN}([\mathrm{R}])[\mathrm{R}]$ & 0.16 & 0.02 & 0.08 \\
\hline$[R] S([R])=0$ & 0.16 & 0.08 & 0.27 \\
\hline$[\mathrm{R}] \mathrm{N}(\mathrm{O}) \mathrm{C}([\mathrm{R}])=\mathrm{O}$ & 0.16 & 0.24 & 0.01 \\
\hline $\mathrm{OC}(=\mathrm{O}) \mathrm{C}=\mathrm{CC}=\mathrm{C}$ & 0.15 & 0.19 & 0 \\
\hline$[\mathrm{R}] \mathrm{C}(=\mathrm{O}) \mathrm{OCOC}=\mathrm{C}$ & 0.15 & 0.03 & 0 \\
\hline$[\mathrm{R}] \mathrm{OCOC}(=\mathrm{O}) \mathrm{C}=\mathrm{C}$ & 0.15 & 0.08 & 0 \\
\hline$[R] N(C([R])=O) C([R])=0$ & 0.15 & 0.14 & 1.48 \\
\hline [R]OCOCO & 0.15 & 0.21 & 0.02 \\
\hline$[\mathrm{R}] \mathrm{OC}(=\mathrm{C}) \mathrm{C}([\mathrm{R}])=\mathrm{O}$ & 0.15 & 0.11 & 0 \\
\hline$[\mathrm{R}] \mathrm{C}([\mathrm{O}-])=\mathrm{O}$ & 0.15 & 0 & 0 \\
\hline$[\mathrm{R}] \mathrm{N}=[\mathrm{Car}]$ & 0.14 & 0.03 & 1.02 \\
\hline$[\mathrm{R}] \mathrm{OCOCOC}=\mathrm{CC}(\mathrm{O})=\mathrm{O}$ & 0.13 & 0.08 & 0 \\
\hline$[\mathrm{R}] \mathrm{C}(=\mathrm{O}) \mathrm{OC}=\mathrm{C}$ & 0.13 & 0.62 & 0.01 \\
\hline $\mathrm{C}=\mathrm{CC} \# \mathrm{CC} \# \mathrm{C}$ & 0.13 & 0.04 & 0 \\
\hline$[\mathrm{R}] \mathrm{OC}=\mathrm{O}$ & 0.12 & 0.05 & 0 \\
\hline
\end{tabular}




\begin{tabular}{|c|c|c|c|}
\hline$[R] S(O)(=O)=O$ & 0.12 & 0.36 & 0.06 \\
\hline$[\mathrm{R}] \mathrm{C}(=\mathrm{O}) \mathrm{C}(=\mathrm{CO}) \mathrm{C}([\mathrm{R}])=\mathrm{O}$ & 0.11 & 0.07 & 0 \\
\hline$[R] N([R]) C N([R])[R]$ & 0.11 & 2.08 & 0.04 \\
\hline$[\mathrm{R}] \mathrm{C}(=\mathrm{O}) \mathrm{OCO}$ & 0.1 & 0.24 & 0 \\
\hline$[\mathrm{R}] \mathrm{OC}(=\mathrm{O}) \mathrm{C}=\mathrm{CC}([\mathrm{R}])=\mathrm{O}$ & 0.09 & 0.05 & 0 \\
\hline$[\mathrm{R}] \mathrm{OCN}([\mathrm{R}]) \mathrm{C}([\mathrm{R}])=\mathrm{O}$ & 0.09 & 0.1 & 0.09 \\
\hline $\mathrm{BrC}=\mathrm{C}$ & 0.09 & 0.01 & 0.02 \\
\hline$[\mathrm{R}] \mathrm{C}(=\mathrm{O}) \mathrm{C}=\mathrm{CC} 1 \mathrm{CO} 1$ & 0.09 & 0.07 & 0 \\
\hline$[\mathrm{R}] \mathrm{N}=\mathrm{C}=\mathrm{S}$ & 0.09 & 0.03 & 0 \\
\hline$[R] N([R]) C=C$ & 0.09 & 0.61 & 0.1 \\
\hline$[\mathrm{R}] \mathrm{C}(=\mathrm{O}) \mathrm{C}=\mathrm{CC}=\mathrm{CC}=\mathrm{C}$ & 0.09 & 0.07 & 0 \\
\hline$[\mathrm{R}][\mathrm{N}+] \#[\mathrm{C}-]$ & 0.09 & 0.04 & 0 \\
\hline $\mathrm{CIC}=\mathrm{C}$ & 0.08 & 0.06 & 0.04 \\
\hline$[R] N([R]) C(=O) C([R])=O$ & 0.08 & 0.07 & 0.08 \\
\hline $\mathrm{C}=\mathrm{CC}=\mathrm{CC}=\mathrm{CC}=\mathrm{C}$ & 0.08 & 0.08 & 0 \\
\hline $\mathrm{C}=\mathrm{CC}(=\mathrm{O}) \mathrm{C} 1 \mathrm{CO} 1$ & 0.08 & 0.1 & 0 \\
\hline$[R] C(=O) C([R])=O$ & 0.08 & 0.03 & 0.01 \\
\hline $\mathrm{C} 1 \mathrm{OC} 1 \mathrm{C} 2 \mathrm{CO} 2$ & 0.08 & 0.03 & 0 \\
\hline$[R] N=C N([R])[R]$ & 0.08 & 0.05 & 0.07 \\
\hline$[\mathrm{R}] \mathrm{OC}(=\mathrm{O}) \mathrm{C}=\mathrm{CC}=\mathrm{CC}=\mathrm{C}$ & 0.08 & 0.1 & 0 \\
\hline$[R] S C=N[R]$ & 0.08 & 0.09 & 0.02 \\
\hline$[\mathrm{R}] \mathrm{C}(=\mathrm{O}) \mathrm{C}=[\mathrm{Car}]$ & 0.08 & 0.14 & 0.02 \\
\hline$[R] S$ & 0.08 & 0.07 & 0.22 \\
\hline$[\mathrm{R}] \mathrm{OC}=\mathrm{N}[\mathrm{R}]$ & 0.07 & 0.11 & 0.02 \\
\hline$[\mathrm{R}] \mathrm{OCSC}=\operatorname{NOS}(\mathrm{O})(=\mathrm{O})=0$ & 0.07 & 0.03 & 0 \\
\hline$[\mathrm{R}] \mathrm{N}([\mathrm{R}]) \mathrm{C}(=\mathrm{O}) \mathrm{C}=\mathrm{NO}$ & 0.07 & 0.01 & 0 \\
\hline$[\mathrm{R}] \mathrm{N}([\mathrm{R}]) \mathrm{C}(=\mathrm{O}) \mathrm{C}(=\mathrm{CO}) \mathrm{C}([\mathrm{R}])=\mathrm{O}$ & 0.07 & 0.1 & 0 \\
\hline$[\mathrm{R}] \mathrm{OC}(=\mathrm{O}) \mathrm{C}=\mathrm{CC} 1 \mathrm{CO} 1$ & 0.07 & 0.03 & 0 \\
\hline$[\mathrm{R}] \mathrm{ON}([\mathrm{R}]) \mathrm{C}([\mathrm{R}])=\mathrm{O}$ & 0.07 & 0.06 & 0.06 \\
\hline $\mathrm{C}=\mathrm{COC}(=\mathrm{O}) \mathrm{C}=\mathrm{C}$ & 0.07 & 0.7 & 0 \\
\hline$[R] l$ & 0.07 & 0.02 & 0.94 \\
\hline$[\mathrm{R}] \mathrm{OC}(\mathrm{O}) \mathrm{C}(\mathrm{O}[\mathrm{R}]) \mathrm{O}[\mathrm{R}]$ & 0.06 & 0.01 & 0 \\
\hline$[\mathrm{R}] \mathrm{ON}=\mathrm{CC}(=\mathrm{O}) \mathrm{N}([\mathrm{R}])[\mathrm{R}]$ & 0.06 & 0.01 & 0.02 \\
\hline $\mathrm{C}=\mathrm{CC}=\mathrm{CC}=\mathrm{O}$ & 0.06 & 0.04 & 0 \\
\hline$[\mathrm{R}] \mathrm{C}(=\mathrm{O}) \mathrm{C}=\mathrm{C}(\mathrm{O}) \mathrm{C}=\mathrm{C}$ & 0.06 & 0.05 & 0 \\
\hline $\mathrm{C}=\mathrm{CC}=[\mathrm{Car}]$ & 0.06 & 0.25 & 0.05 \\
\hline$[R] N([R]) N([R]) C([R])=O$ & 0.06 & 0.1 & 0.03 \\
\hline$[\mathrm{R}] \mathrm{OP}(\mathrm{O})(\mathrm{O})=\mathrm{O}$ & 0.06 & 0.04 & 0.01 \\
\hline$[\mathrm{R}] \mathrm{OC}(=\mathrm{CBr}) \mathrm{C}(\mathrm{Br})=\mathrm{C}$ & 0.05 & 0 & 0 \\
\hline$[\mathrm{R}] \mathrm{OC}(=\mathrm{O}) \mathrm{C}(=\mathrm{C}) \mathrm{C}=\mathrm{C}$ & 0.05 & 0.06 & 0 \\
\hline $\mathrm{OC}(=\mathrm{C}) \mathrm{C}(=\mathrm{O}) \mathrm{C}=\mathrm{C}$ & 0.05 & 0.03 & 0 \\
\hline O[Nar] & 0.05 & 0.07 & 0.01 \\
\hline$[\mathrm{R}] \mathrm{OP}(\mathrm{O})(=\mathrm{O}) \mathrm{O}[\mathrm{R}]$ & 0.05 & 0.03 & 0.01 \\
\hline$[R] S C([R])=0$ & 0.05 & 0.04 & 0.03 \\
\hline$[\mathrm{R}] \mathrm{C}(=\mathrm{O}) \mathrm{C} 1 \mathrm{OC} 1 \mathrm{C}=\mathrm{C}$ & 0.05 & 0.01 & 0 \\
\hline$[\mathrm{R}] \mathrm{C}(=\mathrm{O}) \mathrm{C}(=\mathrm{CO}) \mathrm{C}(=\mathrm{O}) \mathrm{C}=\mathrm{CO}$ & 0.05 & 0.05 & 0 \\
\hline [R]O[Nar] & 0.05 & 0.04 & 0.02 \\
\hline $\mathrm{C}=\mathrm{CC}=\mathrm{CC} \# \mathrm{CC} \# \mathrm{CC}=\mathrm{C}$ & 0.05 & 0.01 & 0 \\
\hline$[\mathrm{R}] \mathrm{N}(\mathrm{C}=\mathrm{C}) \mathrm{C}=\mathrm{O}$ & 0.05 & 0.01 & 0 \\
\hline$[\mathrm{R}] \mathrm{C}([\mathrm{Nar}])=\mathrm{O}$ & 0.05 & 0.03 & 0.17 \\
\hline
\end{tabular}




\begin{tabular}{llrr}
{$[R] O C(=O) C=C O$} & 0.05 & 0.06 & 0 \\
{$[R] S([R])(=O)=O$} & 0.05 & 0.02 & 2.43 \\
{$[R] C(=O) C(=C O) C(O)=O$} & 0.05 & 0.06 & 0 \\
{$[R] O C(O) C 1 C O 1$} & 0.05 & 0.04 & 0 \\
$C=C C=C C=C C=C C=C$ & 0.05 & 0.06 & 0 \\
\hline
\end{tabular}

Table S2. Occurrence (in percentage) of functional groups in natural products from the DNP originated from animals, plants, fungi, bacteria and synthetic molecules (the table may be obtained from the corresponding author upon request as a text file).

\begin{tabular}{|c|c|c|c|c|c|}
\hline$F G$ & animals & plants & fungi & bacteria & synth. \\
\hline $\mathrm{O}[\mathrm{Cal}]$ & 52.81 & 62.42 & 58.26 & 64.49 & 6.22 \\
\hline$[\mathrm{R}] \mathrm{O}[\mathrm{R}]$ & 21.2 & 37.91 & 33.62 & 32.99 & 42.06 \\
\hline $\mathrm{C}=\mathrm{C}$ & 50.35 & 40.82 & 34.22 & 21.69 & 4.04 \\
\hline$[R] O C([R])=0$ & 26.32 & 28.92 & 29.05 & 28.98 & 9.01 \\
\hline$[R] N([R]) C([R])=0$ & 9.79 & 2.67 & 12.96 & 33.51 & 55.97 \\
\hline $\mathrm{O}[\mathrm{Car}]$ & 11.31 & 29.84 & 34.25 & 33.18 & 3.52 \\
\hline [Nar] & 12.89 & 4.84 & 9.03 & 22.42 & 56.33 \\
\hline $\mathrm{O}=[\mathrm{Car}]$ & 7.85 & 13.96 & 18.39 & 20.36 & 14.63 \\
\hline$[R] O C O[R]$ & 10.23 & 26.7 & 6.09 & 23.19 & 2.06 \\
\hline$[R] C([R])=O$ & 11.39 & 16.11 & 19.23 & 15.71 & 3.87 \\
\hline$[R] C(O)=O$ & 10.53 & 8.93 & 14.1 & 22.06 & 4.4 \\
\hline [Oar] & 6.1 & 15.47 & 13.31 & 7.18 & 13.25 \\
\hline$[R] N([R])[R]$ & 3.21 & 6.62 & 1.06 & 6.24 & 21.49 \\
\hline$[\mathrm{R}] \mathrm{Cl}$ & 3.12 & 0.86 & 3.89 & 6.69 & 18.94 \\
\hline$[\mathrm{R}] \mathrm{OC}(=\mathrm{O}) \mathrm{C}=\mathrm{C}$ & 7.44 & 14.47 & 4.4 & 3.19 & 0.3 \\
\hline [Sar] & 0.93 & 0.19 & 0.04 & 4.18 & 17.04 \\
\hline$[\mathrm{R}] \mathrm{C}(=\mathrm{O}) \mathrm{C}=\mathrm{C}$ & 5.38 & 6.31 & 6.5 & 3 & 0.37 \\
\hline$[R] N[R]$ & 3.44 & 1.85 & 0.9 & 5.92 & 7.96 \\
\hline $\mathrm{C}=\mathrm{CC}=\mathrm{C}$ & 5.62 & 1.89 & 3.95 & 8.17 & 0.04 \\
\hline $\mathrm{N}[\mathrm{Cal}]$ & 3.32 & 0.55 & 1.38 & 13.01 & 1.14 \\
\hline$[\mathrm{R}] \mathrm{F}$ & 0.03 & 0.01 & 0.01 & 0.1 & 17.44 \\
\hline$[\mathrm{R}] \mathrm{Br}$ & 6.82 & 0.84 & 0.2 & 0.85 & 6.32 \\
\hline $\mathrm{C} 1 \mathrm{CO} 1$ & 5.46 & 3.5 & 4.02 & 1.55 & 0.08 \\
\hline$[R] S[R]$ & 0.71 & 0.14 & 1.04 & 2.03 & 9.22 \\
\hline$[R] N([R]) S([R])(=O)=O$ & 0.01 & 0 & 0 & 0.01 & 12.72 \\
\hline$[\mathrm{R}] \mathrm{OCO}$ & 1.93 & 2.32 & 2.28 & 5.55 & 0.03 \\
\hline$[\mathrm{R}] \mathrm{C}=\mathrm{O}$ & 2.04 & 2.34 & 3.33 & 2.72 & 0.27 \\
\hline $\mathrm{C}=[\mathrm{Car}]$ & 1 & 0.5 & 0.93 & 0.74 & 4.37 \\
\hline $\mathrm{N}[\mathrm{Car}]$ & 1.73 & 0.13 & 0.47 & 3.06 & 1.87 \\
\hline$[\mathrm{R}] \mathrm{OS}(\mathrm{O})(=\mathrm{O})=\mathrm{O}$ & 4.78 & 0.45 & 0.32 & 1.07 & 0 \\
\hline$[\mathrm{R}] \mathrm{N}(=\mathrm{O})=\mathrm{O}$ & 0.04 & 0.1 & 0.24 & 1.44 & 4.74 \\
\hline$[R] N([R]) C(=O) N([R])[R]$ & 0.17 & 0.05 & 0.04 & 1.52 & 4.51 \\
\hline$[R] N=C(N([R])[R]) N([R])[R]$ & 1.7 & 0.1 & 0.12 & 3.76 & 0.11 \\
\hline $\mathrm{OC}(=\mathrm{O}) \mathrm{C}=\mathrm{C}$ & 0.73 & 1.29 & 2.23 & 1.04 & 0.05 \\
\hline
\end{tabular}




\begin{tabular}{|c|c|c|c|c|c|}
\hline$[R] N([R]) C(=O) C=C$ & 0.95 & 0.65 & 0.56 & 2.02 & 1.1 \\
\hline$[R] O C O C([R])=0$ & 0.27 & 3.27 & 0.76 & 0.29 & 0.02 \\
\hline$[R] O C(=O) N([R])[R]$ & 0.37 & 0.17 & 0.01 & 2.57 & 1.27 \\
\hline $\mathrm{C \# N}$ & 0.33 & 0.16 & 0.03 & 0.41 & 2.95 \\
\hline$[\mathrm{R}] \mathrm{OC}(=\mathrm{O}) \mathrm{C}=\mathrm{CC}=\mathrm{C}$ & 0.52 & 0.42 & 1.12 & 1.16 & 0.01 \\
\hline $\mathrm{C}=\mathrm{CC}=\mathrm{O}$ & 0.72 & 0.98 & 1.15 & 0.23 & \\
\hline $\mathrm{C}=\mathrm{CC}=\mathrm{CC}=\mathrm{C}$ & 0.62 & 0.17 & 0.53 & 1.47 & 0.01 \\
\hline$[R] S([R])(=O)=0$ & 0.2 & 0.03 & 0.07 & 0.03 & 2.43 \\
\hline $\mathrm{C \# C}$ & 1.34 & 0.23 & 0.23 & 0.46 & 0.46 \\
\hline$[R] N(C([R])=O) C([R])=O$ & 0.25 & 0.08 & 0.24 & 0.57 & 1.48 \\
\hline$[\mathrm{R}] \mathrm{N}=[\mathrm{Car}]$ & 0.67 & 0.01 & 0.07 & 0.69 & 1.02 \\
\hline$[R] N([R]) C(=O) C(=C) N([R]) C([R])=O$ & 0.25 & 0.01 & 0.37 & 1.61 & 0.12 \\
\hline$[\mathrm{R}] \mathrm{C}(=\mathrm{O}) \mathrm{C}=\mathrm{CC}=\mathrm{C}$ & 0.24 & 0.28 & 0.87 & 0.94 & 0.01 \\
\hline$[\mathrm{R}] \mathrm{N}(\mathrm{O}) \mathrm{C}([\mathrm{R}])=\mathrm{O}$ & 0.01 & 0.01 & 0.13 & 1.85 & 0.01 \\
\hline$[R] N([R]) C=0$ & 0.81 & 0.14 & 0.05 & 0.91 & 0.04 \\
\hline$[\mathrm{R}] \mathrm{OO}[\mathrm{R}]$ & 1.48 & 0.19 & 0.22 & 0.05 & \\
\hline$[R] N([R]) C N([R]) C([R])=0$ & 0.09 & 0.03 & 1.09 & 0.46 & 0.2 \\
\hline$[\mathrm{R}] \mathrm{OCOCO}[\mathrm{R}]$ & 0.34 & 0.52 & 0.21 & 0.78 & 0.01 \\
\hline$[R] N([R]) C(=O) C=C C=C$ & 0.18 & 0.17 & 0.22 & 1.25 & 0.02 \\
\hline$[\mathrm{R}] \mathrm{N}=\mathrm{C}$ & 0.28 & 0.42 & 0.05 & 0.4 & 0.67 \\
\hline$[\mathrm{R}] \mathrm{C}(=\mathrm{O}) \mathrm{C}=\mathrm{CO}$ & 0.06 & 0.17 & 1.09 & 0.47 & 0.02 \\
\hline $\mathrm{C}=\mathrm{CC}(=\mathrm{O}) \mathrm{C}=\mathrm{C}$ & 0.74 & 0.56 & 0.37 & 0.09 & 0.04 \\
\hline$[\mathrm{R}] \mathrm{N}(\mathrm{CO}) \mathrm{C}([\mathrm{R}])=\mathrm{O}$ & 0.1 & 0.04 & 0.41 & 1.21 & 0.01 \\
\hline$[\mathrm{R}] \mathrm{OC}=\mathrm{CC}([\mathrm{R}])=\mathrm{O}$ & 0.1 & 0.26 & 1.08 & 0.2 & 0.01 \\
\hline$[\mathrm{R}] \mathrm{OC}=\mathrm{C}$ & 0.23 & 0.39 & 0.48 & 0.51 & 0.03 \\
\hline$[\mathrm{R}] \mathrm{OC}(=\mathrm{O}) \mathrm{C} 1 \mathrm{CO} 1$ & 0.99 & 0.43 & 0.13 & 0.08 & 0.01 \\
\hline$[\mathrm{Nar}+]$ & 0.91 & 0.24 & 0.11 & 0.2 & 0.13 \\
\hline $\mathrm{C}=\mathrm{CC} \# \mathrm{C}$ & 1.08 & 0.17 & 0.24 & 0.01 & \\
\hline$[\mathrm{R}][\mathrm{N}+]([\mathrm{R}])([\mathrm{R}])[\mathrm{R}]$ & 0.63 & 0.3 & 0.18 & 0.18 & 0.07 \\
\hline$[R] S S[R]$ & 0.28 & 0.07 & 0.72 & 0.28 & \\
\hline$[R] I$ & 0.33 & 0.04 & 0.01 & 0.03 & 0.94 \\
\hline $\mathrm{S}=[\mathrm{Car}]$ & 0 & 0 & 0.01 & 0.07 & 1.24 \\
\hline$[R] N(C=C) C([R])=O$ & 0.26 & 0.23 & 0.28 & 0.5 & 0.04 \\
\hline$[\mathrm{R}] \mathrm{OCN}([\mathrm{R}])[\mathrm{R}]$ & 0.39 & 0.36 & 0.07 & 0.3 & 0.09 \\
\hline$[R] N([R]) C(=S) N([R])[R]$ & 0.01 & 0 & 0.01 & 0.01 & 1.14 \\
\hline$[\mathrm{R}] \mathrm{OO}$ & 0.65 & 0.41 & 0.08 & 0.02 & \\
\hline$[R] S([R])=0$ & 0.2 & 0.13 & 0.19 & 0.35 & 0.27 \\
\hline $\mathrm{OC}(=\mathrm{O}) \mathrm{C}=\mathrm{CC}=\mathrm{C}$ & 0.05 & 0.08 & 0.5 & 0.49 & \\
\hline$[\mathrm{R}] \mathrm{OC}=\mathrm{CC}(=\mathrm{O}) \mathrm{O}[\mathrm{R}]$ & 0.14 & 0.38 & 0.23 & 0.24 & 0.01 \\
\hline$[\mathrm{R}] \mathrm{C}(=\mathrm{O}) \mathrm{C} 1 \mathrm{CO} 1$ & 0.07 & 0.18 & 0.42 & 0.31 & 0.01 \\
\hline $\mathrm{C}=\mathrm{CC} 1 \mathrm{CO} 1$ & 0.3 & 0.16 & 0.2 & 0.32 & \\
\hline$[\mathrm{R}] \mathrm{SC}=\mathrm{N}[\mathrm{R}]$ & 0.2 & 0 & 0 & 0.74 & 0.02 \\
\hline$[\mathrm{R}] \mathrm{S}(\mathrm{O})(=\mathrm{O})=\mathrm{O}$ & 0.61 & 0.05 & 0.02 & 0.21 & 0.06 \\
\hline$[\mathrm{R}] \mathrm{OCN}([\mathrm{R}]) \mathrm{C}([\mathrm{R}])=\mathrm{O}$ & 0.15 & 0.04 & 0.18 & 0.47 & 0.09 \\
\hline$[R] N([R]) C(=O) C([R])=O$ & 0.22 & 0.01 & 0.04 & 0.57 & 0.08 \\
\hline$[\mathrm{R}][\mathrm{N}+] \#[\mathrm{C}-]$ & 0.47 & 0.01 & 0.04 & 0.38 & \\
\hline$[\mathrm{R}] \mathrm{C}(=\mathrm{O}) \mathrm{C}=\mathrm{CC}([\mathrm{R}])=\mathrm{O}$ & 0.16 & 0.11 & 0.61 & 0.01 & \\
\hline$[\mathrm{R}] \mathrm{OC}(\mathrm{O}[\mathrm{R}]) \mathrm{O}[\mathrm{R}]$ & 0.05 & 0.32 & 0.05 & 0.46 & \\
\hline$[\mathrm{R}] \mathrm{C}([\mathrm{O}-])=\mathrm{O}$ & 0.4 & 0.11 & 0.2 & 0.16 & \\
\hline C\#CC\#C & 0.57 & 0.2 & 0.05 & 0.01 & \\
\hline
\end{tabular}




\begin{tabular}{|c|c|c|c|c|c|}
\hline$[R] N=C N([R])[R]$ & 0.07 & 0.03 & 0.04 & 0.6 & 0.07 \\
\hline $\mathrm{C}=\mathrm{CC}=\mathrm{CC}=\mathrm{CC}=\mathrm{C}$ & 0.09 & 0.02 & 0.17 & 0.52 & 0 \\
\hline$[R] N([R]) N([R]) C([R])=O$ & 0 & 0 & 0.04 & 0.71 & 0.03 \\
\hline$[\mathrm{R}] \mathrm{N} 1 \mathrm{CC}(=\mathrm{O}) \mathrm{N}([\mathrm{R}]) \mathrm{C} 1=\mathrm{O}$ & 0.1 & 0 & 0.01 & 0.01 & 0.64 \\
\hline$[\mathrm{R}] \mathrm{OCOC}=\mathrm{C}$ & 0.01 & 0.2 & 0.28 & 0.27 & 0 \\
\hline$[R] N([R])([R])=0$ & 0.12 & 0.51 & 0.05 & 0.08 & \\
\hline$[R] N([R]) C(=O) C(=C O) C([R])=O$ & 0.03 & 0 & 0.39 & 0.33 & 0 \\
\hline$[\mathrm{R}] \mathrm{OC}=\mathrm{N}[\mathrm{R}]$ & 0.2 & 0.01 & 0.04 & 0.46 & 0.02 \\
\hline$[\mathrm{R}] \mathrm{C}(=\mathrm{O}) \mathrm{C}(\mathrm{O})=\mathrm{C}$ & 0.21 & 0.21 & 0.26 & 0.04 & 0 \\
\hline$[\mathrm{R}] \mathrm{C}(=\mathrm{O}) \mathrm{C}=\mathrm{CC} 1 \mathrm{CO} 1$ & 0.1 & 0.05 & 0.12 & 0.44 & 0 \\
\hline$[\mathrm{R}] \mathrm{C}(=\mathrm{O}) \mathrm{OCO}$ & 0.03 & 0.06 & 0.54 & 0.07 & \\
\hline$[\mathrm{R}] \mathrm{OC}(=\mathrm{O}) \mathrm{C}=\mathrm{CN}=[\mathrm{Car}]$ & 0 & 0 & 0 & 0 & 0.67 \\
\hline$[\mathrm{R}] \mathrm{OP}(\mathrm{O})(\mathrm{O})=\mathrm{O}$ & 0.22 & 0.01 & 0.08 & 0.35 & 0.01 \\
\hline $\mathrm{CIC}=\mathrm{C}$ & 0.2 & 0.04 & 0.06 & 0.3 & 0.04 \\
\hline$[\mathrm{R}] \mathrm{C}(=\mathrm{O}) \mathrm{C}=[\mathrm{Car}]$ & 0 & 0.04 & 0.55 & 0.03 & 0.02 \\
\hline$[\mathrm{R}] \mathrm{N}([\mathrm{R}]) \mathrm{C}(=\mathrm{O}) \mathrm{C}=\mathrm{NO}$ & 0.57 & 0 & 0.02 & 0.05 & \\
\hline$[\mathrm{R}] \mathrm{OC}(=\mathrm{O}) \mathrm{C}=\mathrm{CC}=\mathrm{CC}=\mathrm{C}$ & 0.08 & 0.05 & 0.18 & 0.32 & \\
\hline$[\mathrm{R}] \mathrm{C}(=\mathrm{O}) \mathrm{C}=\mathrm{CC}=\mathrm{CC}=\mathrm{C}$ & 0.19 & 0.05 & 0.28 & 0.1 & \\
\hline$[\mathrm{R}] \mathrm{OCOCOC}=\mathrm{CC}(=\mathrm{O}) \mathrm{O}[\mathrm{R}]$ & 0.05 & 0.55 & 0 & 0 & 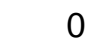 \\
\hline$[\mathrm{R}] \mathrm{N}=\mathrm{C}=\mathrm{S}$ & 0.42 & 0.05 & 0 & 0.13 & 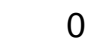 \\
\hline$[R] S$ & 0.11 & 0.07 & 0.03 & 0.17 & 0.22 \\
\hline$[\mathrm{R}] \mathrm{C}(=\mathrm{O}) \mathrm{C}(=\mathrm{CO}) \mathrm{C}(\mathrm{O})=\mathrm{O}$ & 0 & 0 & 0.04 & 0.55 & \\
\hline$[\mathrm{Oar}+]$ & 0.07 & 0.51 & 0.01 & 0 & ( \\
\hline$[\mathrm{R}] \mathrm{N}(\mathrm{O}) \mathrm{C}=\mathrm{O}$ & 0 & 0 & 0.01 & 0.55 & 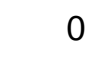 \\
\hline$[R] N([R]) C O$ & 0.09 & 0.23 & 0.01 & 0.21 & 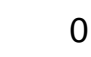 \\
\hline$[\mathrm{R}] \mathrm{C}(=\mathrm{O}) \mathrm{OC}=\mathrm{C}$ & 0.07 & 0.13 & 0.26 & 0.07 & 0.01 \\
\hline $\mathrm{C}=\mathrm{CC}=\mathrm{CC}=\mathrm{CC}=\mathrm{CC}=\mathrm{C}$ & 0.03 & 0.01 & 0.07 & 0.43 & 0 \\
\hline$[\mathrm{Nar}] \mathrm{N}=\mathrm{C}$ & 0 & 0 & 0 & 0.01 & 0.52 \\
\hline O[Nar] & 0 & 0.01 & 0.32 & 0.19 & 0.01 \\
\hline $\mathrm{C}=\mathrm{CC}(=\mathrm{O}) \mathrm{C} 1 \mathrm{CO} 1$ & 0.02 & 0.05 & 0.43 & 0.03 & 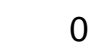 \\
\hline$[R] N([R]) N=C$ & 0 & 0 & 0 & 0 & 0.53 \\
\hline $\mathrm{BrC}=\mathrm{C}$ & 0.41 & 0.05 & 0.01 & 0.03 & 0.02 \\
\hline$[\mathrm{R}] \mathrm{C}(=\mathrm{O}) \mathrm{OCOC}=\mathrm{C}$ & 0.34 & 0.15 & 0.02 & 0 & \\
\hline$[\mathrm{R}] \mathrm{ON}=\mathrm{CC}(=\mathrm{O}) \mathrm{N}([\mathrm{R}])[\mathrm{R}]$ & 0.46 & 0 & 0.02 & 0.01 & 0.02 \\
\hline$[\mathrm{R}] \mathrm{C}(=\mathrm{O}) \mathrm{C}(=\mathrm{CO}) \mathrm{C}([\mathrm{R}])=\mathrm{O}$ & 0.02 & 0.11 & 0.09 & 0.29 & \\
\hline$[R] N(N=C) C([R])=O$ & 0 & 0 & 0 & 0.18 & 0.3 \\
\hline$[R] N([R]) C(=O) N([R]) C([R])=O$ & 0.05 & 0 & 0.01 & 0.02 & 0.39 \\
\hline$[\mathrm{R}] \mathrm{N}([\mathrm{R}]) \mathrm{C}(=\mathrm{O}) \mathrm{C}=\mathrm{CC}=\mathrm{CC}=\mathrm{C}$ & 0.06 & 0 & 0.07 & 0.34 & \\
\hline$[R] N([R]) C(=S) N([R]) C([R])=O$ & 0 & 0 & 0 & 0 & 0.47 \\
\hline$[\mathrm{R}] \mathrm{SC}([\mathrm{R}])=\mathrm{O}$ & 0.06 & 0.02 & 0.02 & 0.33 & 0.03 \\
\hline$[\mathrm{R}] \mathrm{OCOCO}$ & 0.06 & 0.17 & 0.15 & 0.06 & 0.02 \\
\hline$[\mathrm{R}] \mathrm{SC} 1 \mathrm{CC}(=\mathrm{O}) \mathrm{N} 1 \mathrm{C}(=\mathrm{C}) \mathrm{C}(\mathrm{O})=\mathrm{O}$ & 0 & 0 & 0.07 & 0.38 & 0.01 \\
\hline$[R] N([R]) C=C C([R])=O$ & 0.01 & 0.02 & 0.02 & 0.12 & 0.28 \\
\hline$[\mathrm{R}] \mathrm{N}([\mathrm{R}]) \mathrm{C}(=\mathrm{O}) \mathrm{C}(=\mathrm{O}) \mathrm{N}([\mathrm{R}])[\mathrm{R}]$ & 0.03 & 0 & 0.01 & 0 & 0.41 \\
\hline $\mathrm{OC}(=\mathrm{O}) \mathrm{C}=\mathrm{CC}=\mathrm{CC}=\mathrm{C}$ & 0 & 0 & 0.1 & 0.35 & \\
\hline$[R] N([R]) C N([R])[R]$ & 0.24 & 0.1 & 0.05 & 0.02 & 0.04 \\
\hline $\mathrm{C}=\mathrm{CC} \# \mathrm{CC} \# \mathrm{C}$ & 0.21 & 0.14 & 0.09 & 0.01 & \\
\hline$[\mathrm{R}] \mathrm{C}(=\mathrm{O}) \mathrm{C}(\mathrm{Cl})=[\mathrm{Car}]$ & 0 & 0 & 0.44 & 0 & , \\
\hline$[\mathrm{R}] \mathrm{C}([\mathrm{Nar}])=\mathrm{O}$ & 0.02 & 0.04 & 0.07 & 0.11 & 0.17 \\
\hline$[\mathrm{R}] \mathrm{OC}(=\mathrm{CBr}) \mathrm{C}(\mathrm{Br})=\mathrm{C}$ & 0.41 & 0 & 0 & 0 & \\
\hline
\end{tabular}




\begin{tabular}{|c|c|c|c|c|c|}
\hline$[\mathrm{R}] \mathrm{OP}(\mathrm{O})(=\mathrm{O}) \mathrm{O}[\mathrm{R}]$ & 0.2 & 0.02 & 0.02 & 0.15 & 0.01 \\
\hline$[\mathrm{R}] \mathrm{N}(\mathrm{C}=\mathrm{C}) \mathrm{C}=\mathrm{O}$ & 0.28 & 0.01 & 0.01 & 0.1 & \\
\hline $\mathrm{C}=\mathrm{CC}=\mathrm{CC}=\mathrm{CC}=\mathrm{CC}=\mathrm{CC}=\mathrm{CC}=\mathrm{C}$ & 0.01 & 0.01 & 0.02 & 0.36 & \\
\hline $\mathrm{ON}=\mathrm{C}$ & 0.03 & 0 & 0.01 & 0.27 & 0.08 \\
\hline$[R] N([R]) C(=O) C(O)=C C([R])=O$ & 0 & 0 & 0 & 0 & 0.37 \\
\hline$[\mathrm{R}] \mathrm{N}([\mathrm{R}]) \mathrm{C}=\mathrm{C}$ & 0.12 & 0.1 & 0.03 & 0.01 & 0.1 \\
\hline$[\mathrm{R}] \mathrm{OC}=\mathrm{O}$ & 0.07 & 0.14 & 0.1 & 0.04 & \\
\hline $\mathrm{O}=[\mathrm{Nar}]$ & 0.01 & 0.02 & 0.04 & 0.22 & 0.06 \\
\hline$[R] O C(=C) C([R])=O$ & 0.1 & 0.18 & 0.06 & 0.01 & \\
\hline$[\mathrm{R}] \mathrm{OCOCOC}=\mathrm{C}$ & 0 & 0.35 & 0 & 0 & \\
\hline$[\mathrm{R}] \mathrm{C}(=\mathrm{O}) \mathrm{C}(\mathrm{O})=\mathrm{O}$ & 0.01 & 0.02 & 0.1 & 0.22 & \\
\hline$[\mathrm{R}] \mathrm{OC}(=\mathrm{O}) \mathrm{C}(\mathrm{O}[\mathrm{R}])=\mathrm{CC}=\mathrm{C}$ & 0 & 0 & 0 & 0.35 & \\
\hline $\mathrm{C}=\mathrm{CC}=\mathrm{CC}=\mathrm{CC}=\mathrm{CC}=\mathrm{CC}=\mathrm{CC}=\mathrm{CC}=\mathrm{CC}=\mathrm{C}$ & 0.16 & 0.02 & 0.01 & 0.16 & \\
\hline$[\mathrm{R}] \mathrm{OC}=\mathrm{CN}([\mathrm{R}]) \mathrm{C}([\mathrm{R}])=\mathrm{O}$ & 0 & 0 & 0 & 0.34 & \\
\hline $\mathrm{C}=\mathrm{CC}=\mathrm{CC}=\mathrm{CC}=\mathrm{CC}=\mathrm{CC}=\mathrm{CC}=\mathrm{CC}=\mathrm{CC}=\mathrm{CC}=\mathrm{CC}=\mathrm{C}$ & 0.06 & 0.02 & 0.01 & 0.25 & \\
\hline$[\mathrm{R}] \mathrm{N}([\mathrm{R}]) \mathrm{C}=[\mathrm{Car}]$ & 0.08 & 0.01 & 0.04 & 0.02 & 0.19 \\
\hline$[\mathrm{R}] \mathrm{OC}(=\mathrm{O}) \mathrm{C}=\mathrm{CC}([\mathrm{R}])=\mathrm{O}$ & 0.05 & 0.09 & 0.17 & 0.02 & \\
\hline$[R] N([R]) S(=O)(=O) N([R])[R]$ & 0 & 0 & 0 & 0 & 0.33 \\
\hline$[R] S C N([R]) C([R])=O$ & 0 & 0 & 0.05 & 0.07 & 0.2 \\
\hline$[R] \mathrm{ON}([\mathrm{R}]) \mathrm{C}([\mathrm{R}])=\mathrm{O}$ & 0.01 & 0.07 & 0.11 & 0.07 & 0.06 \\
\hline$[\mathrm{R}] \mathrm{OCOCN}([\mathrm{R}]) \mathrm{C}([\mathrm{R}])=\mathrm{O}$ & 0.15 & 0 & 0 & 0.17 & \\
\hline$[\mathrm{R}] \mathrm{OC}(=\mathrm{O}) \mathrm{N}([\mathrm{R}]) \mathrm{CO}$ & 0.01 & 0.02 & 0 & 0.29 & \\
\hline$[R] N(O)[R]$ & 0.16 & 0.01 & 0.03 & 0.1 & 0.01 \\
\hline $\mathrm{C}=\mathrm{CC}=\mathrm{CC}=\mathrm{O}$ & 0.06 & 0.06 & 0.14 & 0.05 & \\
\hline $\mathrm{C}=\mathrm{COC}=\mathrm{C}$ & 0.02 & 0.01 & 0.27 & 0 & \\
\hline$[\mathrm{R}] \mathrm{ON}([\mathrm{R}])[\mathrm{R}]$ & 0.13 & 0.01 & 0.01 & 0.02 & 0.13 \\
\hline$[\mathrm{R}] \mathrm{OC}(=\mathrm{O}) \mathrm{C}=\mathrm{CN}([\mathrm{R}])[\mathrm{R}]$ & 0 & 0.22 & 0 & 0 & 0.08 \\
\hline$[\mathrm{R}] \mathrm{O}[\mathrm{Nar}]$ & 0.03 & 0.04 & 0.19 & 0.01 & 0.02 \\
\hline$[R] S S S[R]$ & 0.04 & 0.01 & 0.18 & 0.06 & \\
\hline $\mathrm{OC}(=\mathrm{C}) \mathrm{C}(\mathrm{O})=\mathrm{O}$ & 0.01 & 0.02 & 0.2 & 0.05 & \\
\hline$[\mathrm{R}] \mathrm{C}(=\mathrm{O}) \mathrm{C} 1 \mathrm{OC} 1 \mathrm{C}([\mathrm{R}])=\mathrm{O}$ & 0.01 & 0.01 & 0.04 & 0.22 & \\
\hline $\mathrm{C} 1 \mathrm{OC} 1 \mathrm{C} 2 \mathrm{CO} 2$ & 0.11 & 0.08 & 0.02 & 0.07 & \\
\hline$[\mathrm{R}] \mathrm{OCOC}(=\mathrm{O}) \mathrm{C}=\mathrm{C}$ & 0.04 & 0.2 & 0.02 & 0.01 & \\
\hline$[R] \operatorname{OCN}([R]) C(=N[R]) N([R])[R]$ & 0.18 & 0 & 0 & 0.09 & \\
\hline$[\mathrm{R}] \mathrm{OC}(=\mathrm{O}) \mathrm{C}(=\mathrm{C}) \mathrm{C}([\mathrm{R}])=\mathrm{O}$ & 0.08 & 0.01 & 0.15 & 0.03 & \\
\hline $\begin{array}{l}{[\mathrm{R}] \mathrm{N} 1 \mathrm{CC}(=\mathrm{O}) \mathrm{N}([\mathrm{R}]) \mathrm{C}(=\mathrm{C}) \mathrm{C} 1=\mathrm{O}} \\
\mathrm{C}=\mathrm{CC}=\mathrm{CC}=\mathrm{CC}=\mathrm{CC}=\mathrm{CC}=\mathrm{CC}=\mathrm{CC}=\mathrm{CC}=\mathrm{CC}=\mathrm{CC}=\mathrm{CC}=\end{array}$ & 0.02 & 0 & 0.18 & 0.06 & \\
\hline C & 0.01 & 0 & 0.02 & 0.23 & \\
\hline$[\mathrm{R}] \mathrm{N}(\mathrm{O}) \mathrm{C}(=\mathrm{O}) \mathrm{C}=\mathrm{C}$ & 0 & 0 & 0.18 & 0.08 & \\
\hline$[\mathrm{R}] \mathrm{C}(=\mathrm{O}) \mathrm{OCOCOC}([\mathrm{R}])=\mathrm{O}$ & 0.24 & 0.02 & 0 & 0 & \\
\hline $\mathrm{C}=\mathrm{CC}=\mathrm{CC}(=\mathrm{O}) \mathrm{C}=\mathrm{C}$ & 0.19 & 0.02 & 0.03 & 0.01 & \\
\hline $\mathrm{OC}=\mathrm{CC}(\mathrm{O})=\mathrm{O}$ & 0.16 & 0 & 0.08 & 0.01 & \\
\hline$[\mathrm{R}] \mathrm{OC}(=\mathrm{O}) \mathrm{C}=\mathrm{CO}$ & 0.01 & 0.04 & 0.05 & 0.15 & \\
\hline$[\mathrm{R}] \mathrm{ON}=\mathrm{C}$ & 0.03 & 0 & 0.01 & 0 & 0. \\
\hline$[R] N(C=C) C(=O) C(=C) N([R]) C([R])=O$ & 0.04 & 0 & 0.02 & 0.18 & \\
\hline$[\mathrm{R}] \mathrm{OC}(=\mathrm{O}) \mathrm{C}=\mathrm{COP}(=\mathrm{O})(\mathrm{O}[\mathrm{R}]) \mathrm{O}[\mathrm{R}]$ & 0 & 0 & 0 & 0.23 & \\
\hline $\mathrm{BrC}=\mathrm{CC}=\mathrm{C}$ & 0.12 & 0.01 & 0 & 0.1 & \\
\hline$[R] P(O)(O)=O$ & 0.02 & 0 & 0 & 0.2 & 0.01 \\
\hline$[\mathrm{R}] \mathrm{OS}([\mathrm{R}])(=\mathrm{O})=\mathrm{O}$ & 0.01 & 0 & 0 & 0.01 & 0.2 \\
\hline$[\mathrm{R}] \mathrm{N}([\mathrm{R}]) \mathrm{C}(=\mathrm{O}) \mathrm{C}(=\mathrm{O}) \mathrm{C}=\mathrm{CO}$ & 0 & 0 & 0 & 0 & 0.22 \\
\hline$[\mathrm{R}] \mathrm{OC}(=\mathrm{O}) \mathrm{C}=\mathrm{CC} 1 \mathrm{CO} 1$ & 0.01 & 0.07 & 0.1 & 0.04 & \\
\hline
\end{tabular}




\begin{tabular}{|c|c|c|c|c|c|}
\hline $\mathrm{C}=\mathrm{CC}=\mathrm{CC}=\mathrm{CC}=\mathrm{CC}=\mathrm{CC}=\mathrm{CC}=\mathrm{CC}=\mathrm{CC}=\mathrm{CC}=\mathrm{C}$ & 0.09 & 0.02 & 0.01 & 0.1 & 0 \\
\hline$[R] N([R])[N a r]$ & 0 & 0 & 0 & 0.04 & 0.18 \\
\hline $\mathrm{C}=\mathrm{C}=\mathrm{C}$ & 0.09 & 0.01 & 0.12 & 0 & 0 \\
\hline $\mathrm{BrC}(\mathrm{Br})=\mathrm{C}$ & 0.19 & 0.01 & 0.01 & 0 & 0 \\
\hline$[R] S S S S[R]$ & 0 & 0.01 & 0.19 & 0.01 & 0 \\
\hline$[\mathrm{R}] \mathrm{OC}(=\mathrm{O}) \mathrm{C}=\mathrm{CC}(\mathrm{O})=\mathrm{O}$ & 0 & 0.01 & 0.07 & 0.13 & 0 \\
\hline$[R] N([R]) C=S$ & 0 & 0 & 0 & 0.13 & 0.08 \\
\hline$[\mathrm{R}] \mathrm{N}=\mathrm{N}([\mathrm{R}])=[\mathrm{Car}]$ & 0 & 0 & 0.01 & 0.2 & 0 \\
\hline$[\mathrm{R}] \mathrm{N}(\mathrm{C}(=\mathrm{O}) \mathrm{C}=\mathrm{CC}=\mathrm{CC}=\mathrm{C}) \mathrm{C}(=\mathrm{CO}) \mathrm{C}([\mathrm{R}])=\mathrm{O}$ & 0 & 0 & 0 & 0.21 & 0 \\
\hline$[\mathrm{R}] \mathrm{OC}(=\mathrm{O}) \mathrm{C}(=\mathrm{C}) \mathrm{C}=\mathrm{C}$ & 0.02 & 0.04 & 0.11 & 0.04 & 0 \\
\hline$[\mathrm{R}] \mathrm{SCN}([\mathrm{R}])[\mathrm{R}]$ & 0.02 & 0 & 0 & 0.15 & 0.04 \\
\hline$[\mathrm{R}] \mathrm{OC}(=\mathrm{O}) \mathrm{C}=\mathrm{CN}([\mathrm{R}]) \mathrm{C}(=\mathrm{O}) \mathrm{N}([\mathrm{R}])[\mathrm{R}]$ & 0 & 0 & 0 & 0 & 0.21 \\
\hline$[R] N=C(N([R])[R]) N([R]) C O$ & 0.14 & 0 & 0 & 0.06 & 0 \\
\hline$[\mathrm{R}] \mathrm{C}(=\mathrm{O}) \mathrm{C}=\mathrm{C}(\mathrm{O}) \mathrm{C}=\mathrm{CC}=\mathrm{C}$ & 0 & 0 & 0.2 & 0 & 0 \\
\hline $\mathrm{CIC}=\mathrm{CC}=\mathrm{C}$ & 0.02 & 0.01 & 0.13 & 0.04 & 0 \\
\hline$[\mathrm{R}] \mathrm{N}=\mathrm{CO}$ & 0 & 0.01 & 0 & 0.01 & 0.18 \\
\hline$[\mathrm{R}] \mathrm{OC}(=\mathrm{O}) \mathrm{O}[\mathrm{R}]$ & 0 & 0.02 & 0.12 & 0.04 & 0.02 \\
\hline$[\mathrm{R}] \mathrm{C}(=\mathrm{O}) \mathrm{N}=[\mathrm{Car}]$ & 0 & 0 & 0 & 0 & 0.19 \\
\hline$[\mathrm{R}] \mathrm{OC}(=\mathrm{C}) \mathrm{C}(=\mathrm{O}) \mathrm{O}[\mathrm{R}]$ & 0.01 & 0.01 & 0.11 & 0.06 & 0 \\
\hline$[R] N(C N([R]) C([R])=O) C([R])=O$ & 0.01 & 0.03 & 0.11 & 0.02 & 0.02 \\
\hline$[\mathrm{R}] \mathrm{C}(=\mathrm{O}) \mathrm{C}=\mathrm{CC}(\mathrm{O})=\mathrm{O}$ & 0.02 & 0.03 & 0.1 & 0.03 & 0 \\
\hline$[\mathrm{R}] \mathrm{OCOCOC}=\mathrm{CC}(\mathrm{O})=\mathrm{O}$ & 0 & 0.18 & 0 & 0 & 0 \\
\hline$[\mathrm{R}] \mathrm{OCS}[\mathrm{R}]$ & 0.01 & 0.01 & 0 & 0.15 & 0.01 \\
\hline$[R] N(C O C([R])=O) C([R])=O$ & 0 & 0 & 0 & 0.17 & 0 \\
\hline$[R] N(C([R])=O) S([R])(=O)=O$ & 0 & 0 & 0 & 0.01 & 0.16 \\
\hline$[\mathrm{R}] \mathrm{C}(=\mathrm{O}) \mathrm{C}(\mathrm{Br})=\mathrm{C}$ & 0.15 & 0 & 0.01 & 0.01 & 0 \\
\hline$[\mathrm{R}] \mathrm{OC}(=\mathrm{O}) \mathrm{C}(=\mathrm{C}) \mathrm{N}([\mathrm{R}]) \mathrm{C}([\mathrm{R}])=\mathrm{O}$ & 0 & 0 & 0.02 & 0.14 & 0.01 \\
\hline$[\mathrm{R}] \mathrm{OC}(=\mathrm{CBr}) \mathrm{C}(\mathrm{Br})=\mathrm{COCON}=\mathrm{CC}(=\mathrm{O}) \mathrm{N}([\mathrm{R}])[\mathrm{R}]$ & 0.17 & 0 & 0 & 0 & 0 \\
\hline$[\mathrm{R}] \mathrm{OC}(=\mathrm{O}) \mathrm{C}=\mathrm{CC}=\mathrm{CC}=\mathrm{CC}=\mathrm{CC}=\mathrm{C}$ & 0 & 0 & 0 & 0.16 & 0 \\
\hline $\mathrm{OS}(=\mathrm{O})(=\mathrm{O}) \mathrm{OC}=\mathrm{C}$ & 0.16 & 0 & 0 & 0 & 0 \\
\hline$[\mathrm{R}] \mathrm{C}(=\mathrm{O}) \mathrm{C}=\mathrm{CC}=\mathrm{CC}=\mathrm{CC}=\mathrm{C}$ & 0.1 & 0 & 0.02 & 0.04 & 0 \\
\hline$[R] N([R]) C(C([R])=O)=C(C(O)=O) C([R])=O$ & 0 & 0 & 0 & 0.16 & 0 \\
\hline $\begin{array}{l}{[\mathrm{R}] \mathrm{C}(=\mathrm{O}) \mathrm{C}(=\mathrm{CC}=[\mathrm{Car}]) \mathrm{C}(\mathrm{O})=\mathrm{O}} \\
{[\mathrm{R}] \mathrm{C}(=\mathrm{O}) \mathrm{C}=\mathrm{CC}=\mathrm{CC}=\mathrm{CC}=\mathrm{CC}=\mathrm{CC}=\mathrm{CC}=\mathrm{CC}=\mathrm{CC}=\mathrm{CC}=}\end{array}$ & 0.05 & 0 & 0.11 & 0 & 0 \\
\hline $\mathrm{CC}=\mathrm{CC}=\mathrm{C}$ & 0.02 & 0 & 0.01 & 0.13 & 0 \\
\hline$[\mathrm{R}] \mathrm{N} 1 \mathrm{C}(\mathrm{O}) \mathrm{OCC} 1=\mathrm{O}$ & 0 & 0.01 & 0.15 & 0 & 0 \\
\hline$[R] N([R]) \operatorname{COC}([R])=O$ & 0 & 0.03 & 0.01 & 0.1 & 0.01 \\
\hline$[\mathrm{R}] \mathrm{OC}=\mathrm{CC}(=\mathrm{O}) \mathrm{C}=\mathrm{C}$ & 0 & 0.05 & 0.1 & 0 & 0 \\
\hline$[\mathrm{R}] \mathrm{OCOO}[\mathrm{R}]$ & 0.14 & 0.01 & 0 & 0 & 0 \\
\hline$[\mathrm{R}] \mathrm{OC}(=\mathrm{O}) \mathrm{C}=\mathrm{CN}([\mathrm{R}]) \mathrm{C}=\mathrm{CC}([\mathrm{R}])=\mathrm{O}$ & 0 & 0 & 0 & 0 & 0.15 \\
\hline$[R] N=C 1 N([R]) C C(=O) N 1[R]$ & 0.15 & 0 & 0 & 0 & 0 \\
\hline$[\mathrm{R}] \mathrm{C}(=\mathrm{O}) \mathrm{C}=\mathrm{CC}=\mathrm{CC}=\mathrm{CC}=\mathrm{CC}=\mathrm{CC}=\mathrm{CC}=\mathrm{CC}=\mathrm{C}=\mathrm{C}$ & 0.15 & 0 & 0 & 0 & 0 \\
\hline$[\mathrm{R}] \mathrm{OC}=\mathrm{CC}(=\mathrm{O}) \mathrm{N}([\mathrm{R}])[\mathrm{R}]$ & 0.01 & 0.01 & 0 & 0.12 & 0.01 \\
\hline$[\mathrm{R}] \mathrm{C}(=\mathrm{O}) \mathrm{OCOCO}$ & 0.13 & 0.01 & 0 & 0 & 0 \\
\hline$[R] N(C([R])=O) C(=C) C(O)=O$ & 0 & 0 & 0.03 & 0.11 & 0 \\
\hline $\mathrm{BrC}=\mathrm{CC}(\mathrm{Br})=\mathrm{C}$ & 0.12 & 0 & 0.01 & 0.01 & 0 \\
\hline$[R] S C(=N[R]) N([R]) C([R])=O$ & 0 & 0 & 0 & 0 & 0.14 \\
\hline $\begin{array}{l}{[\mathrm{R}] \mathrm{OC}=[\mathrm{Car}]} \\
{[\mathrm{R}] \mathrm{C}(=\mathrm{O}) \mathrm{C}=\mathrm{CC}=\mathrm{CC}=\mathrm{CC}=\mathrm{CC}=\mathrm{CC}=\mathrm{CC}=\mathrm{CC}=\mathrm{CC}=\mathrm{CC}=}\end{array}$ & 0 & 0.02 & 0.11 & 0.01 & 0 \\
\hline $\mathrm{CC}=\mathrm{C}$ & 0.01 & 0.01 & 0 & 0.12 & 0 \\
\hline$[R] N([R]) C(=O) C(C([R])=O)=C(O) C=C$ & 0 & 0 & 0.02 & 0.11 & 0 \\
\hline
\end{tabular}




\begin{tabular}{|c|c|c|c|c|c|}
\hline$[R] N([R]) C(=O) C(C([R])=O)=C(O) C=C C=C$ & 0 & 0 & 0.03 & 0.1 & 0 \\
\hline$[R] S C(=N C([R])=O) N([R])[R]$ & 0 & 0 & 0 & 0 & 0.13 \\
\hline$[\mathrm{R}] \mathrm{OP}([\mathrm{O}-])(=\mathrm{O}) \mathrm{O}[\mathrm{R}]$ & 0.11 & 0 & 0.01 & 0.01 & 0 \\
\hline$[R] S C=C(N([R]) C([R])=O) C(O)=O$ & 0 & 0 & 0 & 0.13 & 0 \\
\hline $\mathrm{C}=\mathrm{CC}=\mathrm{CC} 1 \mathrm{OC} 1 \mathrm{C}=\mathrm{C}$ & 0 & 0 & 0 & 0.13 & 0 \\
\hline $\begin{array}{l}{[\mathrm{R}] \mathrm{OC}(=\mathrm{O}) \mathrm{C}=\mathrm{CC}=\mathrm{CC}=\mathrm{CC}=\mathrm{C}} \\
\mathrm{C}=\mathrm{CC}=\mathrm{CC}=\mathrm{CC}=\mathrm{CC}=\mathrm{CC}=\mathrm{CC}=\mathrm{CC}=\mathrm{CC}=\mathrm{CC}=\mathrm{CC}=\mathrm{CC}=\end{array}$ & 0.01 & 0.01 & 0.01 & 0.1 & 0 \\
\hline $\mathrm{CC}=\mathrm{C}$ & 0 & 0 & 0.02 & 0.1 & 0 \\
\hline$[R] S C(=N[R]) N([R])[R]$ & 0 & 0 & 0 & 0 & 0.12 \\
\hline$[R] N=N[R]$ & 0 & 0 & 0.01 & 0 & 0.11 \\
\hline $\mathrm{OC}(=\mathrm{O}) \mathrm{C}=[\mathrm{Car}]$ & 0 & 0 & 0.12 & 0 & 0 \\
\hline $\mathrm{BrC}=\mathrm{CC \# C}$ & 0.11 & 0 & 0.01 & 0 & 0 \\
\hline$[\mathrm{R}] \mathrm{OCOP}(\mathrm{O})(=\mathrm{O}) \mathrm{O}[\mathrm{R}]$ & 0.01 & 0 & 0 & 0.11 & 0 \\
\hline$[R] P(O)([R])=O$ & 0 & 0 & 0 & 0.11 & 0 \\
\hline $\mathrm{C}=\mathrm{COC}(=\mathrm{O}) \mathrm{C}=\mathrm{C}$ & 0 & 0.1 & 0 & 0.01 & 0 \\
\hline$[R] S C(=N[R]) C(=C) N([R]) C([R])=O$ & 0 & 0 & 0 & 0.11 & 0 \\
\hline$[\mathrm{R}][\mathrm{N}+]([\mathrm{R}])=[\mathrm{Car}]$ & 0.11 & 0 & 0 & 0 & 0 \\
\hline $\mathrm{BrC}=\mathrm{CC} \# \mathrm{CC}=\mathrm{C}$ & 0.11 & 0 & 0 & 0 & 0 \\
\hline$[\mathrm{R}] \mathrm{N}([\mathrm{Nar}]) \mathrm{C}([\mathrm{R}])=\mathrm{O}$ & 0 & 0 & 0 & 0 & 0.11 \\
\hline$[\mathrm{R}] \mathrm{OCN}([\mathrm{R}]) \mathrm{N}=\mathrm{C}$ & 0 & 0 & 0 & 0 & 0.11 \\
\hline$[\mathrm{R}] \mathrm{OCSC}=\operatorname{NOS}(\mathrm{O})(=\mathrm{O})=\mathrm{O}$ & 0 & 0.1 & 0 & 0 & 0 \\
\hline$[\mathrm{R}] \mathrm{C}(=\mathrm{O}) \mathrm{C}=\mathrm{CC}=\mathrm{C}(\mathrm{O}) \mathrm{C}=\mathrm{C}$ & 0 & 0 & 0.1 & 0 & 0 \\
\hline $\mathrm{OC}(=\mathrm{O}) \mathrm{C \# C}$ & 0.1 & 0 & 0 & 0 & 0 \\
\hline [R]SC\#N & 0.1 & 0 & 0 & 0 & 0 \\
\hline $\begin{array}{l}{[R] N(C([R])=O) S(O)(=O)=O} \\
{[R] N([R]) C(=O) C(=C) N([R]) C(=O) C(=C) N([R]) C([R])=}\end{array}$ & 0 & 0 & 0 & 0.1 & 0 \\
\hline 0 & 0 & 0 & 0 & 0.1 & 0 \\
\hline
\end{tabular}

